\title{
Comparación de metodologías ágiles y procesos de desarrollo de software mediante un instrumento basado en CMMI
}

\author{
Mapping agile methodologies and software development processes using a CMMI based \\ instrument.
}

\author{
Jaime Andrés Britto Montoya \\ Ingeniero de Sistemas, Universidad Autónoma de Manizales, Manizales, Colombia \\ Correo-e: jaimeandresbritto@gmail.com
}

\begin{abstract}
Resumen- En la literatura científica se pueden encontrar comparaciones entre metodologías ágiles y CMMI, estas se encuentran generalmente limitadas a unas pocas áreas de proceso. Este artículo presenta la construcción de un instrumento de comparación que toma como referencia el cubrimiento obtenido sobre las prácticas específicas de CMMI, estableciendo así un marco común sobre el cual se pueden comparar metodologías ágiles y procesos de desarrollo de software. Aquí se evaluaron y compararon Scrum, XP e Iconix y un proceso de desarrollo de software de una empresa del eje cafetero colombiano, demostrando la funcionalidad del instrumento como método de evaluación y validación.
\end{abstract}

Palabras clave- comparación de metodologías, CMMI, ICONIX, metodologías ágiles, SCRUM, XP.

Abstract- In scientific literature, comparisons can be found between CMMI and agile methodologies; generally limited to a few areas of process. This paper discusses the construction of an instrument of comparison taken as reference coverage obtained on the specific practices of CMMI, thus establishing a common framework on which to compare agile methodologies and software development processes. Scrum, XP and Iconix were evaluated and compared, and a process of software development of an enterprise of the Colombian coffee region assessed, demonstrating the functionality of the instrument as a method of evaluation and validation.

Key Word - agile methodologies, CMMI, ICONIX, SCRUM, XP, methodologies mapping.

\section{INTRODUCCIÓN}

Para la implementación de procesos de desarrollo de software en pequeñas empresas debe realizarse un ajuste a estos procesos o metodologías que tengan en cuenta la cultura y el entorno de la empresa [1], además para estas empresas de menor escala realizar un proceso de evaluación y mejora tradicional es muy costoso y complejo. De aquí deriva la necesidad de un instrumento de fácil uso que permita no solo la evaluación del proceso de desarrollo actual de las empresas sino también la comparación contra un modelo base como CMMI y contra metodologías de desarrollo ágil.

La relación de SCRUM y CMMI en las áreas de PP, PMC, SAM, IPM, RSKM, QPM fueron analizadas en el trabajo de [2]. Allí se analizó el propósito de cada práctica específica de dichas áreas y se contrastó con las prácticas definidas en SCRUM, estableciendo en qué medida la práctica de CMMI se encontraba satisfecha. Luego este estudio fue profundizado analizando la correspondencia de SCRUM y XP pero solo en las áreas de proceso PP, PMC y REQM por [3] en este caso el método usado para analizar la correspondencia con CMMI fue mediante una evaluación a nivel de subprácticas.

Este método de comparación puede ser usado no solo para contrastar metodologías sino también para medir el estado inicial de un proceso de desarrollo de software y el estado final luego de un proceso de mejora. En este artículo se muestra la construcción y uso de un instrumento basado parcialmente en CMMI y su uso en estos tres campos: Comparación de métodos, medición inicial y final para procesos de desarrollo de software.

\section{CONTENIDO}

\section{A. Instrumento de comparación}

1) Calificación frente a CMMI

El instrumento es de desarrollo propio, basado en las experiencias de comparación de prácticas ágiles frente CMMI-DEV a nivel de subprácticas propuesto por [3] en su tesis de máster. Los estudios mencionados [2] [3] coinciden en el uso de 3 criterios para calificar el cubrimiento de cada práctica de CMMI (ver Tabla 1).

\begin{tabular}{|c|c|}
\hline \multicolumn{1}{|c|}{ Calificación } & \multicolumn{1}{c|}{ Criterio } \\
\hline No soportada (NS) & $\begin{array}{l}\text { La práctica no es soportada por } \\
\text { las prácticas de la metodología. }\end{array}$ \\
\hline
\end{tabular}




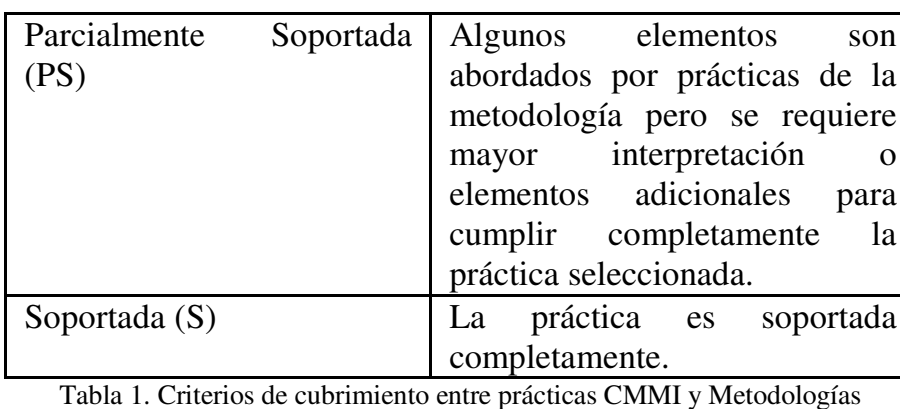

Tabla 1. Criterios de cubrimiento entre prácticas CMMI y Metodologías ágiles.

Este sistema de calificación fue afinado luego de observar que un análisis más detallado de calificación basado en las subprácticas permitía definir mejor las diferencias y fortalezas entre las diferentes metodologías, quedando finalmente con las calificaciones que muestra la Tabla 3 .

\begin{tabular}{|c|c|}
\hline Calificación & Criterio \\
\hline No soportada (NS) & $\begin{array}{l}\text { La práctica no es soportada por } \\
\text { las prácticas de la metodología }\end{array}$ \\
\hline $\begin{array}{l}\text { Parcialmente Soportada en } \\
\text { menor medida (PS-) }\end{array}$ & 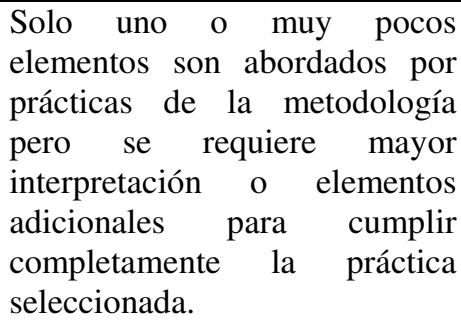 \\
\hline $\begin{array}{ll}\begin{array}{l}\text { Parcialmente } \\
\text { (PS) }\end{array} & \text { Soportada } \\
\end{array}$ & 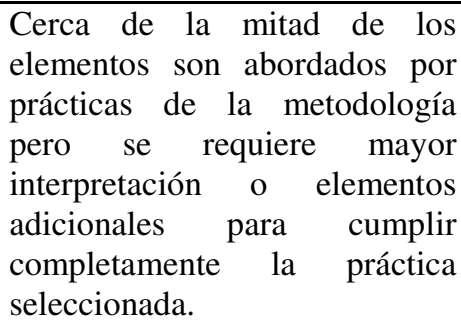 \\
\hline $\begin{array}{l}\text { Parcialmente Soportada en } \\
\text { gran medida }(\mathrm{PS}+)\end{array}$ & $\begin{array}{l}\text { La mayoría de los elementos } \\
\text { son abordados por prácticas de } \\
\text { la metodología pero se requiere } \\
\text { mayor interpretación o } \\
\text { elementos adicionales para } \\
\text { cumplir completamente la } \\
\text { practica seleccionada. }\end{array}$ \\
\hline Soportada (S) & $\begin{array}{l}\text { La práctica es soportada } \\
\text { completamente. }\end{array}$ \\
\hline
\end{tabular}

Tabla 2. Calificaciones ajustadas a 5 niveles de detalle.

Scrum, XP e Iconix fueron comparados con cada subpráctica asignando la calificación "OK" en caso de considerarse que se cumple con la subpráctica o "X" en caso negativo, esta calificación definirá con mayor precisión la calificación para la práctica específica de CMMI correspondiente.
A continuación, en la Tabla 3 se puede observar un ejemplo de esta comparación con SCRUM en el área de proceso Administrar Los Requerimientos (REQM):

\begin{tabular}{|l|l|l|}
\hline Práctica específica & $\begin{array}{l}\text { 1) SP1.1 Comprenden los } \\
\text { requerimientos (requisitos) de los } \\
\text { proveedores sobre el significado de } \\
\text { dichos requerimientos. }\end{array}$ \\
\hline $\begin{array}{l}\text { Calificación de } \\
\text { subprácticas }\end{array}$ & $\begin{array}{l}\text { OK 1. Establecer criterios para } \\
\text { distinguir a los proveedores } \\
\text { apropiados de requisitos. }\end{array}$
\end{tabular}
$\mathrm{X}$ 2. Establecer criterios objetivos para la evaluación y aceptación de los requisitos.

$\mathrm{X}$ 3. Analizar los requisitos para asegurar que se cumplen los criterios establecidos.

OK 4. Alcanzar una comprensión de los requisitos con los proveedores de requisitos para que los participantes del proyecto puedan comprometerse con ellos.

Calificación práctica PS (Parcialmente soportada) especifica

Observación

Durante el pre-game se definen los roles incluyendo al propietario del producto y los clientes representativos, estos proveen los requerimientos y apoyan al equipo proporcionando detalles sobre los mismos.

Requiere de interpretación, el cliente debe involucrarse durante todo el ciclo de vida y no solo al principio y puede agregar nuevas funcionalidades a medida que descubre nuevos requerimientos.

Tabla 3. Ejemplo de comparación de Scrum frente a CMMI.

Casi todas las prácticas específicas de todas las áreas de proceso fueron analizadas de esta forma, algunas que claramente no eran soportadas o no eran del alcance de la metodología no fueron analizadas a nivel de subpráctica dada su irrelevancia.

I. Calificación Cuantitativa

Una vez obtenida la calificación a nivel de subprácticas estas calificaciones se convierten a calificaciones cuantitativas que pueden ser promediadas con el fin de calcular un porcentaje de cubrimiento, estas calificaciones numéricas pueden ser fácilmente comparadas permitiendo contrastar el nivel de cubrimiento de los métodos o procesos evaluados entre sí. Para este fin se usa el instrumento propuesto por [4] que será denominado en adelante instrumento UAM.

Con base en la evaluación por prácticas específicas realizadas se generó la homologación correspondiente con el fin de poder comparar la evaluación mediante el instrumento UAM y 
las matrices de comparación frente a CMMI de cada metodología como se puede observar en la Tabla 4.

\begin{tabular}{|l|l|l|}
\hline \multicolumn{2}{|c|}{ Calificación } \\
\hline \multicolumn{2}{|l|}{ Instrumento de caracterización } & Matriz de cumplimiento \\
\hline 0 & Nunca & No Soportada \\
\hline 1 & Casi nunca & Parcialmente Soportada - \\
\hline 2 & Algunas veces & Parcialmente Soportada \\
\hline 3 & Muchas veces & Parcialmente Soportada + \\
\hline 4 & Siempre & Soportada \\
\hline
\end{tabular}

Las calificaciones de cada metodología frente a CMMI realizadas en el numeral anterior fueron transcritas al instrumento de la UAM con el fin de lograr una calificación numérica que pudiera ser comparada con la evaluación realizada al proceso de desarrollo de software de la empresa evaluada.

La Tabla 5 muestra la conversión de las calificaciones alfanuméricas a numéricas según la homologación propuesta para el área de proceso PMC con respecto a XP. En la tercera columna se puede observar la evaluación obtenida por ejemplo para la práctica específica 1.1 de la meta específica SG1 como Parcialmente soportada (PS), lo que se traduce en una calificación numérica de 2 . Luego se puede calcular el porcentaje de cumplimiento de las metas específicas y del área de proceso teniendo en cuenta el número de prácticas especificas totales y el puntaje total obtenido, aplicando la siguiente fórmula ${ }^{1}$ :

\section{PorcentajeCubrimiento

$$
=\frac{\sum \text { Puntajeobtenido }}{\text { PuntajeMáximo } \times \text { NumeroTotalPrácticas }}
$$

\begin{tabular}{|l|l|l|l|l|}
\hline $\begin{array}{l}\text { MONITOREO Y CONTROL DE PROYECTOS } \\
\text { (PMC) }\end{array}$ & $55 \%$ \\
\hline $\begin{array}{l}\text { SG1 MONITOREAR EL PROYECTO CONTRA } \\
\text { EL PLAN (PMC) }\end{array}$ & $43 \%$ \\
\hline SP 1,1 & 2 & & PS & \\
\hline SP 1,2 & 4 & & S & \\
\hline SP 1,3 & 0 & & NS & \\
\hline SP 1,4 & 0 & & NS & \\
\hline SP 1,5 & 0 & & NS & \\
\hline SP 1,6 & 2 & & PS & \\
\hline SP 1,7 & 4 & & S & \\
\hline 7 & 12 & & & \\
\hline
\end{tabular}

${ }^{1}$ El puntaje máximo corresponde al valor 4, que es la puntuación máxima posible permitida por el instrumento UAM.

\begin{tabular}{|l|l|l|l|l|}
\hline \multicolumn{4}{|l|}{ SG2 ADMINISTRAN ACCION CORRECTIVA } & $83 \%$ \\
HASTA EL CIERRE (PMC) & \\
\hline SP 2,1 & 4 & & S & \\
\hline SP 2,2 & 4 & & S & \\
\hline SP 2,3 & 2 & & PS & \\
\hline 3 & 10 & & & \\
\hline 10 & 22 & & & \\
\hline
\end{tabular}

Tabla 5. Homologación de calificaciones y cálculo del porcentaje de cubrimiento de la metodología frente a CMMI para el área PMC con respecto a la metodología XP

\section{B. Comparación XP, SCRUM e ICONIX}

Usando el instrumento mencionado se compararon XP, SCRUM e ICONIX frente a las prácticas específicas de CMMI obteniendo los resultados que se pueden observar en la Tabla 6, en tonos rojos se observan cubrimientos inferiores al $30 \%$, en tonos amarillos entre 30 y $50 \%$ y en tonos verdes cubrimientos superiores.

\begin{tabular}{|c|l|c|c|c|}
\hline Nivel & Área de Proceso & SCRUM & XP & ICONIX \\
\hline 2 & 1. REQM & $80 \%$ & $80 \%$ & $90 \%$ \\
\hline 2 & 2. PP & $68 \%$ & $43 \%$ & $14 \%$ \\
\hline 2 & 3. PMC & $88 \%$ & $55 \%$ & $0 \%$ \\
\hline 2 & 4. SAM & $0 \%$ & $0 \%$ & $0 \%$ \\
\hline 2 & $5 . \mathrm{MA}$ & $38 \%$ & $38 \%$ & $0 \%$ \\
\hline 2 & 6. PPQA & $13 \%$ & $50 \%$ & $13 \%$ \\
\hline 2 & 7. CM & $0 \%$ & $100 \%$ & $0 \%$ \\
\hline 3 & $8 . \mathrm{REQD}$ & $93 \%$ & $93 \%$ & $83 \%$ \\
\hline 3 & $9 . \mathrm{TS}$ & $0 \%$ & $31 \%$ & $44 \%$ \\
\hline 3 & $10 . \mathrm{PI}$ & $0 \%$ & $61 \%$ & $53 \%$ \\
\hline 3 & $11 . \mathrm{VER}$ & $0 \%$ & $72 \%$ & $69 \%$ \\
\hline 3 & $12 . \mathrm{VAL}$ & $80 \%$ & $90 \%$ & $55 \%$ \\
\hline 3 & $13 . \mathrm{OPF}$ & $0 \%$ & $0 \%$ & $0 \%$ \\
\hline 3 & $14 . \mathrm{OPD}$ & $0 \%$ & $0 \%$ & $0 \%$ \\
\hline 3 & $15 . \mathrm{OT}$ & $0 \%$ & $14 \%$ & $0 \%$ \\
\hline 3 & $16 . \mathrm{IPM}$ & $58 \%$ & $33 \%$ & $0 \%$ \\
\hline 3 & $17 . \mathrm{RSKM}$ & $7 \%$ & $36 \%$ & $0 \%$ \\
\hline 3 & $18 . \mathrm{DAR}$ & $0 \%$ & $0 \%$ & $0 \%$ \\
\hline 4 & $19 . \mathrm{OPP}$ & $0 \%$ & $0 \%$ & $0 \%$ \\
\hline 4 & $20 . \mathrm{QPM}$ & $0 \%$ & $0 \%$ & $0 \%$ \\
\hline 5 & $21 . \mathrm{OPM}$ & $0 \%$ & $0 \%$ & $0 \%$ \\
\hline 5 & $22 . \mathrm{CAR}$ & $0 \%$ & $0 \%$ & $0 \%$ \\
\hline
\end{tabular}

Tabla 6. Resumen cubrimiento porcentual por área de proceso de cada metodología 
Se puede observar un cubrimiento nulo en los niveles 4 y 5 por las prácticas ágiles frente a CMMI, lo que confirma lo que estudios previos muestran [5] [6] [7].

Scrum demuestra su fortaleza principalmente en las áreas REQD, PMC, REQM, VAL. También se observa que XP tiene un cubrimiento mayor de CMMI en los niveles $2^{2}$ y 3 . Los únicos procesos de nivel 3 que XP no cubre son OPF, OPD y DAR proceso que tienen un enfoque organizacional y quedan fuera del alcance de XP.

Finalmente si solo se analiza este indicador de cubrimiento parece demostrar que ICONIX no agrega nuevos elementos a la mezcla de Scrum y XP, mostrando solo un mayor cubrimiento en las áreas de proceso REQM y TS. Así Scrum e ICONIX parecen ser más complementarios que una mezcla de este último frente a XP, ya que Scrum está más enfocado a gestión mientras que tanto XP como ICONIX se concentran en la construcción y desarrollo del producto.

\section{Evaluación inicial de un proceso de desarrollo de software}

Se realizó la evaluación del proceso de desarrollo de software de una empresa de la región que desarrolla su propio software en casa. Las evaluaciones fueron realizadas con el coordinador de desarrollo del proceso quién calificó cada punto usando la tabla de valores sugerido por el instrumento, basado en su experiencia y conocimiento de los procesos y su aplicación específica en dos de los proyectos más grandes y riesgosos abordados hasta el momento.

El resumen del resultado obtenido con el instrumento evaluado detalladamente a nivel de subprácticas se puede observar en la Figura 1.

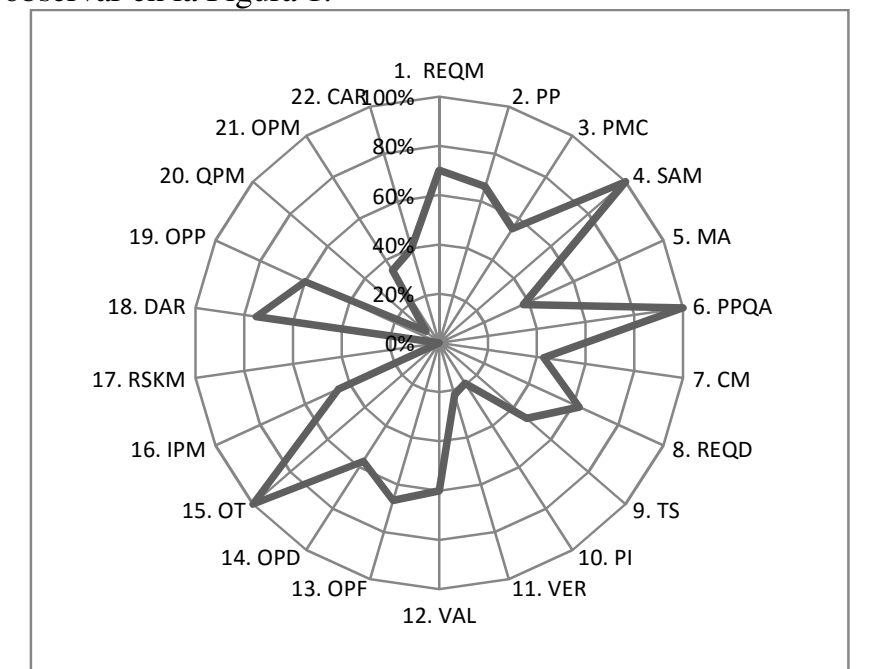

Figura 1. Resultado porcentual cumplimiento por área de proceso evaluación inicial del área de desarrollo de $S W$

${ }^{2}$ Ninguno de los métodos analizados en este estudio tienen un cubrimiento del área de proceso SAM debido al enfoque de estos métodos ágiles al desarrollo en casa en contraste a la práctica de contratación de proveedores.
Tres áreas de proceso fueron identificadas como de un mayor grado de cubrimiento (mayor a 90\%): Administración de contratos con proveedores (SAM), Aseguramiento de la calidad del proceso y del producto (PPQA), Entrenamiento Organizacional (OT).

Otros 6 de obtuvieron los cubrimientos más bajas (menor a 40\%): Administración de Riesgos (RSKM), Administración Cuantitativa de Proyectos (QPM), Integración de Productos (PI), Verificación (VER), Gestión del rendimiento de la organización (OPM), Medición y Análisis (MA).

La mayoría de fortalezas encontradas son derivadas de la aplicación del sistema de calidad implementado en la empresa y de la gestión del área administrativa tanto del proceso como de la organización, áreas de proceso como la administración de proveedores y entrenamiento organizacional tienen procesos bien establecidos y controlados desde la dirección; por otro lado las áreas de aseguramiento de la calidad del proceso y del producto y de validación, son fuertemente apoyadas por el área de auditoría y control interno de la institución. Solo el área de desarrollo de requerimientos resalta como una fortaleza del área técnica encargada del mantenimiento y desarrollo de proyectos de software.

Las debilidades en cambio se encuentran en su mayoría en áreas relacionadas con la gestión y la medición (Monitoreo y Control de Proyectos, Definición de Procesos Organizacionales, Administración Integrada de Proyectos, Medición y Análisis, Administración de Riesgos, Administración Cuantitativa de Proyectos, Gestión del rendimiento de la organización.) y algunas relacionadas con la correcta documentación o registro (Análisis de Causas y Resolución, Administración de Configuraciones), y solo evidencia un área relacionada con elementos técnicos (Integración de Productos).

\section{Comparación entre metodologías y el proceso de software}

Una vez se realizada la calificación del cubrimiento de las prácticas ágiles y de las prácticas aplicadas en el proceso de software de la empresa mencionada frente a CMMI es posible realizar una comparación entre las metodologías y el proceso actual. En la Figura 2 se demuestra como la comparación a simple vista puede aportar información sobre los posibles beneficios para el proceso de desarrollo de la empresa en cuestión de la aplicación de prácticas de XP en las áreas de CM, REQD, VAL e incluso en RSKM. Iconix parece apoyar igualmente las áreas de REQM y REQD mientras que SCRUM aportaría mejoras en PMC y en IPM. 


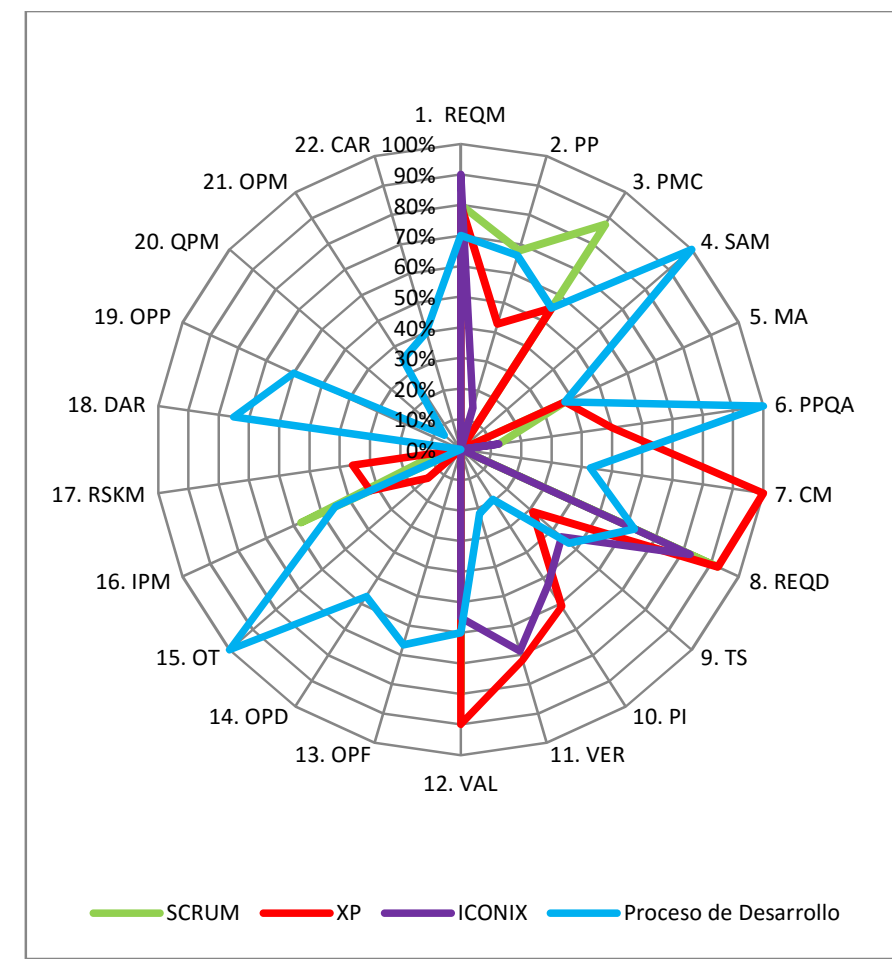

Figura 2. Comparación radial del cubrimiento de CMMI por las metodologías y el proceso actual del ADSCR

\section{E. Evaluación final del proceso}

Luego de realizar un proceso de mejora ${ }^{3}$ es posible medir nuevamente el proceso de desarrollo de software de la empresa, este instrumento puede utilizarse nuevamente con el fin de obtener una nueva calificación de cubrimiento frente a las prácticas propuestas por CMMI y de esta manera conocer el porcentaje de mejora en comparación con el estado inicial.

En la Figura 3 se puede observar en color verde las áreas donde se obtuvo un mejor cubrimiento de las prácticas propuestas por CMMI en contraste al estado inicial del proceso de desarrollo de la empresa.

Las áreas de proceso de Administración de Requerimientos (REQM), Monitoreo y Control de Proyectos (PMC), Administración de Configuraciones (CM), Desarrollo de Requerimientos (REQD), Integración de Productos (PI), Verificación (VER), Validación (VAL), Administración Integrada de Proyectos (IPM) y Administración de Riesgos (RSKM).

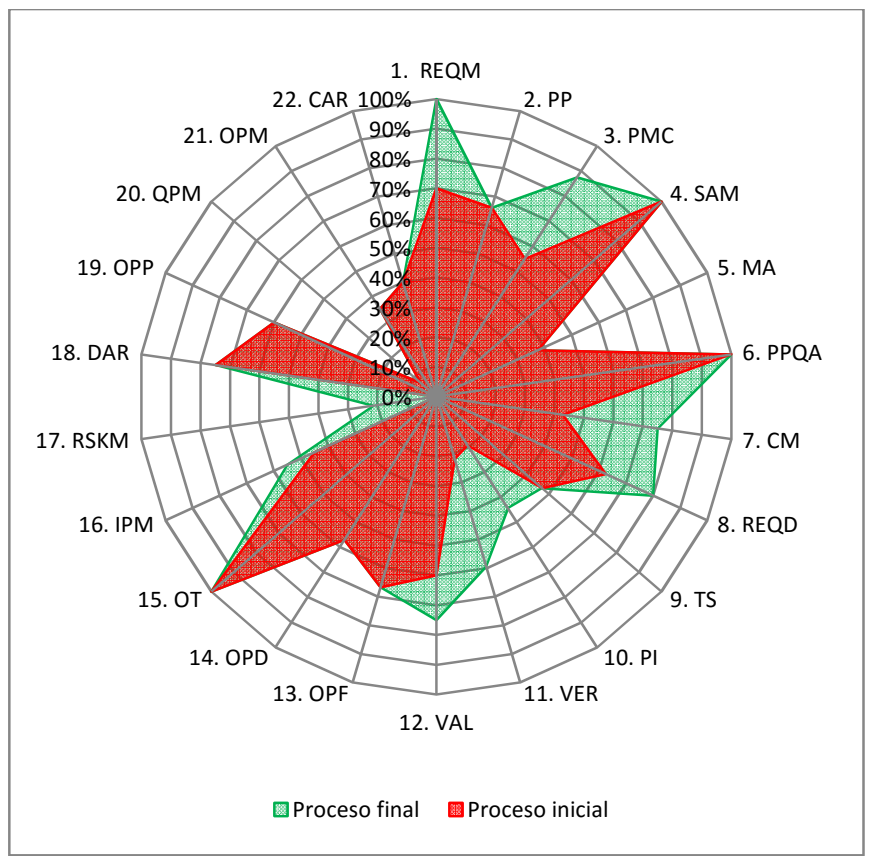

Figura 3. Comparación del proceso de desarrollo inicial y final luego del proceso de mejora.

\section{CONCLUSIONES}

Este es uno de los estudios de mayor cubrimiento hasta la fecha, usa los 5 niveles de CMMI e incluye la comparación total con 3 prácticas ágiles. Generalmente los estudios de este tipo comparan entre 1 y 2 prácticas y solo en un pequeño número de áreas de proceso.

El método elegido para realizar el mapeo de CMMI frente a prácticas ágiles puede extenderse fácilmente a cualquier método o proceso, y da una visión realista del cubrimiento según lo propuesto en CMMI, esto permite fácilmente reconocer las áreas de fortaleza del método o prácticas que se toman como candidatos.

Al realizar un análisis cruzado con mapeos realizados por diferentes autores de las mismas prácticas puede observarse diferencias entre los puntos de vista, lo que muestra que es tal vez imposible no tener cierto nivel de subjetividad al realizar análisis de estas características.

Esta subjetividad se marca con mayor intensidad si se tiene en cuenta que el propio manual técnico de CMMI se dan pautas para la aplicación de practica ágiles que indican que las prácticas especificas podrían verse cubiertas sin la necesidad de seguir las al pie de la letra las indicaciones propuestas por las prácticas o por las sub-prácticas respectivas. Este caso se acentúa más en el caso de prácticas con enfoques menos tradicionalistas como es el caso de la gestión de riesgos en XP.

Al usar una base de comparación tan completa como CMMI, es posible obtener una matriz de mapeo donde todas las prácticas estudiadas demuestran sus fortalezas y debilidades frente al primero, lo que permite tener una visión completa de cómo se pueden complementar unas a otras según las áreas de proceso.

\footnotetext{
${ }^{3}$ Este proceso escapa del alcance de este artículo.
} 


\section{REFERENCIAS}

[1]. S. Ambler and M. Lines, Disciplined agile delivery: A practitioner's guide to agile software delivery in the enterprise. 2012.

[2]. A. Marcal, B. de Freitas, F. Furtado Soares and A. Belchior, 'Mapping CMMI project management process areas to SCRUM practices', 2007, pp. 13--22.

[3]. J. Díaz Fernández, 'Estudio sobre la correspondencia entre prácticas CMMI y prácticas ágiles y su aplicación en Pymes', 2009.

[4]. I. Peralta, 'Caracterización del nivel de desarrollo de software de las empresas de manizales', 2007.

[5]. M. Paulk, 'Extreme programming from a CMM perspective', Software, IEEE, vol 18, iss 6, pp. 19-26, 2001.

[6]. M. Fritzsche, P. Keil and others, 'Agile methods and CMMI: compatibility or conflict?', e-Informatica, vol 1, iss 1, pp. 9--26, 2007.

[7]. G, T. omani and H. Zulzalil, 'Compatibility of Agile Software Development Methods and CMMI.', Indian Journal of Science I\& Technology, vol 6, iss 8, 2013.

[8]. W. Wake, Extreme programming explored. 2002.

[9]. D. Rosenberg and M. Stephens, Use Case Driven Object Modeling with UMLTheory and Practice. 2007.

[10]. L. Scott, R. Jeffery, L. Carvalho, J. D'ambra and P. Rutherford, 'Practical software process improvement-the IMPACT project', 2001, pp. 182-189.

[11]. J. Rumbaugh, G. Booch and I. Jacobson, El lenguaje unificado de modelado: manual de referencia. 2000.

[12]. F. Pino, J. Vidal, F. García and M. Piattini, 'Modelo para la Implementación de Mejora de Procesos en Pequeñas Organizaciones Software.', 2007, pp. 326--335.

[13]. M. Kulpa and K. Johnson, Interpreting the CMMI (R): A Process Improvement Approach. 2004.

[14]. I. Jacobson, G. Booch and J. Rumbaugh, El proceso unificado de desarrollo de software. 2000.
[15]. G. Booch, J. Rumbaugh and I. Jacobson, 'The unified modeling language user guide', Reading, PA: Addison-Wesley, 1999.

[16]. K. Beck and C. Andres, Extreme programming explained: embrace change. 2004.

[17]. D. Ahern, A. Clouse and R. Turner, CMMI distilled: a practical introduction to integrated process improvement. 2004.

[18]. C. Team, 'CMMItextregistered for Development, Version 1.3, Improving processes for developing better products and services', no. CMU/SEI-2010-TR-033. Software Engineering Institute, 2010.

[19]. M. Fayad, M. Laitinen and R. Ward, 'Thinking objectively: software engineering in the small', Communications of the ACM, vol 43, iss 3, pp. 115--118, 2000.

[20]. K. Lukasiewicz and J. Miler, 'Improving agility and discipline of software development with the Scrum and CMMI', Software, IET, vol 6, iss 5, pp. 416--422, 2012.

[21]. J. Diaz, J. Garbajosa and J. Calvo-Manzano, 'Mapping CMMI level 2 to scrum practices: An experience report', Springer, pp. 93--104, 2009.

[22]. R. Pressman, '. Mc Grah-Hill, 7a. edició, 2010', Ingeniería del software, un enfoque práctico.

[23]. M. Phillips and S. Shrum, 'Which CMMI Model Is for You? I SEI Digital Library', Resources.sei.cmu.edu, 2011. [Online]. Available: http://resources.sei.cmu.edu/library/asset-

view.cfm?assetID=28807. [Accessed: 25- May2014].

[24]. C. Sibbald, 'epf/general/getting_started', The Eclipse Foundation open source community., 2006. [Online]. Available: http://www.eclipse.org/epf/general/An_Introduction to_EPF.zip. [Accessed: 25- May- 2014].

[25]. Omg.org, 'Software and Systems Process Engineering Meta-Model Specification', 2008. [Online]. Available: http://www.omg.org/spec/SPEM/2.0/. [Accessed: 25May- 2014].

[26]. Eclipse Process Framework, 'EPF Practices Library Downloads', 2014. [Online]. Available: http://www.eclipse.org/epf/downloads/praclib/praclib _downloads.php. [Accessed: 25- May- 2014]. 